\title{
Developing of computer models of modern measuring instruments for a remote virtual laboratory
}

\author{
Dmitry Gubsky ${ }^{1, *}$, Irina Ivanova ${ }^{1}$, and Anatoly Kleschenkov ${ }^{1}$ \\ ${ }^{1}$ Southern Federal University, Faculty of Physics, 344090, Zorge 5, Rostov-on-Don, Russia
}

\begin{abstract}
The study introduces the approach of creating a virtual laboratory for performing laboratory works with remote access. The proposed virtual lab can be used for distance learning and is an extension of the functionality of the previously created virtual workshop. This virtual laboratory with remote access is an alternative to laboratory works performed with real experimental equipment and provides an opportunity to gain skills in operation with measuring microwave equipment without giving classes in educational laboratories. The results of testing virtual lab works with remote access in educational radiophysics laboratories are presented.
\end{abstract}

\section{Introduction}

Educational programs of many technical specialties include practical classes in educational laboratories. As a rule, such laboratories should be equipped with modern experimental equipment and facilities. However, for many areas of knowledge the cost of experimental laboratory equipments is very high, which requires a lot of money to equip laboratories and limits the number of laboratory setups. In addition, there may be some other reasons why real laboratories are unavailable to students (e.g., overloaded with other experiments, budgets shortage, time constantans, old / outdated equipment, maintenance problems, etc.)

At the same time modern information technologies offer a lot of prospects to get relevant training by virtualizing the educational process. One of them is to create virtual laboratory units [1-3]. Virtual laboratories can significantly change the teaching mode and, as a result, reduce expenses on equipment, overcome time and space limitations and lack of experimental resources. Currently, a sufficiently large number of virtual engineering laboratories in various fields of physics and electronics have been developed [2, 4-7].

There is a great variety of works in radiophysics [3, 7, 8-12]. However, of a particular interest are lab works with the interface of virtual laboratory units being almost the same to that of their real prototypes [4, 12], among which there are several facilities simulating laboratory equipment in terms of the concept and principles of conducting measurements [4].

In the last decade, distance learning which requires laboratory workshops to be carried out remotely has gained a lot of popularity in higher education [5-7, 12-15]. In this respect,

* Corresponding author: $\underline{\text { ds } @ \text { s,sfedu.ru }}$ 
the use of traditional hands-on laboratories is becoming impossible. On the other hand, remote laboratories can be included into engineering courses to eliminate the limitations of traditional laboratories, such as scheduling, cost of equipment and location. Therefore, the use of remote laboratories can also be considered as an intermediate stage between simulations and traditional laboratories.

Modern educational technologies involving computer modeling and multimedia can enhance the interaction between students and teachers and improve the efficiency of the educational process. This increases the role of students in the educational process and raises motivation as students have more freedom in choosing the place and time to perform various tasks. The use of Internet technologies in the computer-based education forms the concept of distance learning due to which students can have online access to courses at any time and from any place, reducing the cost of educational resources.

Considering the above, the development and implementation of remote laboratory works equally aimed at studying the observed physical processes, as well as mastering the techniques of working with modern measuring equipment is in high demand today. Taking into account the educational process, laboratory works with remote access to real laboratory equipment [7, 11, 14-15] and virtual works performed remotely [12] are of practical interest. Such work can be implemented as a web application or a client-server model using a thin client on the client/user side. It means that a small application that does not require computing resources is installed and executed on the user computer. At the same time, simulated tools and devices should have a "visual appearance" similar to that of their "real" counterparts and provide access to "real" controls. This work extends the previous research [16] and is aimed at solving the problem of creating a virtual laboratory with remote access.

The proposed client-server architecture of the virtual laboratory makes it possible to create easily configurable and expandable virtual laboratories designed to study microwave devices and gain skills in iperation with real microwave measuring equipment.

\section{Problem statement}

The main disadvantage of autonomous laboratory works is that they require full software installation on a user computer, and, as a result, when making changes (new releases, improvements, extensions, etc.), the user has to update existing software. In addition, all software must be developed taking into account a wide range of operating systems used by the users on their devices, including mobile ones.

Therefore, the main objective of this work was to create a virtual laboratory with remote access and minimize the amount of software installed on the user side. To implement this task, first it is necessary to develop the concept of splitting up an autonomous virtual measuring device into two parts (client-server) and further develop a protocol for their interaction, providing data transfer between them.

With this approach, the virtual laboratory software with remote access consists of two parts. The first, which is a server component, is installed on a remote computer and includes the main software modules: a database of collected laboratory works, numerical data (binary files) with the results of calculations and real-life experiments for further processing and displaying on the screens of measuring devices, as well as server modules of virtual models software.

And the second part is the client one, which is installed on the user's device and is mainly responsible for displaying the interface of the measuring device and outputting the necessary information. It should be noted that the user interface of the measuring instruments is its photo.

According to the implementation of the proposed concept, it is supposed to develop an approach of splitting the previously developed autonomous laboratory into two units [16- 
17] and create a protocol for data exchange between these units in a client-server architecture.

\section{Virtual device model}

The previously created model of the autonomous device [18] can be divided into two parts according to its functionality. One of them is responsible for processing and preparing data for their visualization on the screen, and the other makes possible to display data and the user interface of the model.

Therefore, it makes sense to allocate the part that is responsible for the implementation of the user interface to the client modules, which will make it independent from the rest of the software and the users only need to install it on their devices (including mobile ones) running any OS (Windows, Android).

Let us consider a simulation of a measuring device with remote access using a vector network analyzer ZVA 40 by Rohde \& Schwarz, whose computer interface is shown in Fig. 1.

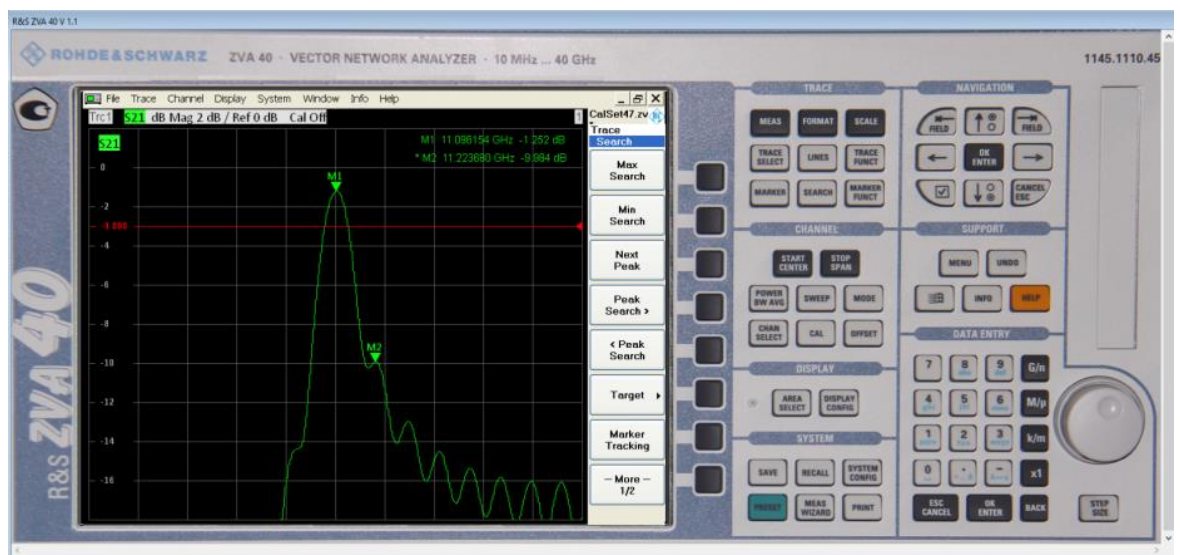

Fig. 1. Interface of vector network analyzer.

This device has a wide range of functional features. However, while being operated, the number of frequently used functions is quite small but enough to carry out all the necessary measurements. Therefore, to gain the basic skills necessary in operation with a vector network analyzer, it is enough to implement a limited number of functions. This computer model allows the user to use buttons for selecting the frequency sweep range (Start/Center and Stop/Span); modes for selecting measured parameters (Meas), formatting the displayed results on the screen (Scale); various modes of marker functions for measurement convenience (Marker, Search, Marker Funct), as well as some functions for data entry, selection unit measurement, etc. To select the desired function and perform its functionality it is necessary to use the mouse and "press" the corresponding device key and (or) select the corresponding control of the on-screen menu.

When a "left mouse button" click event is detected, a handler that determines which grid of the device is pressed on the grid is called. When a user clicks on the allowed button, the corresponding method is called. All methods can be divided into two types: with redrawing the characteristics of the device under study on its screen and without it.

If it is not necessary to redraw the characteristics, for example, setting markers, searching extremums on the displayed characteristic and so on, then the actions performed do not require any access to the server. In this case, all the functionality is implemented by internal methods of the created "Vector network analyzer" class and is executed on the client. For example, when a user selects the maximum value search function on the 
displayed curve (Marker Funct/Max Search), it calls the method that searches the maximum value in the S-parameters set previously received from the server and stored in a special array with index as frequency. After finding the maximum value, the corresponding method of "drawing" the marker with the given coordinates and values displayed on the screen is called. All other internal methods of the device are implemented with the same algorithm.

If it is necessary to redraw the characteristics of the device under study (for example, the user has changed the range of displayed frequencies or output parameters), the server is accessed for the required data.

In this case, we follow some certain rules: the client sends a request to the server and waits for a response, which contains a set of necessary S-parameters to get the amplitudefrequency response of the device under study. After obtaining and processing the response, a special array is created, the index of which is the frequency value of the specified sweep range. This particular array is further used by all internal methods of "Vector network analyzer" class.

The interaction process of the various parts of the device models included in the laboratory work "band-pass filter" is schematically shown in Fig. 2.

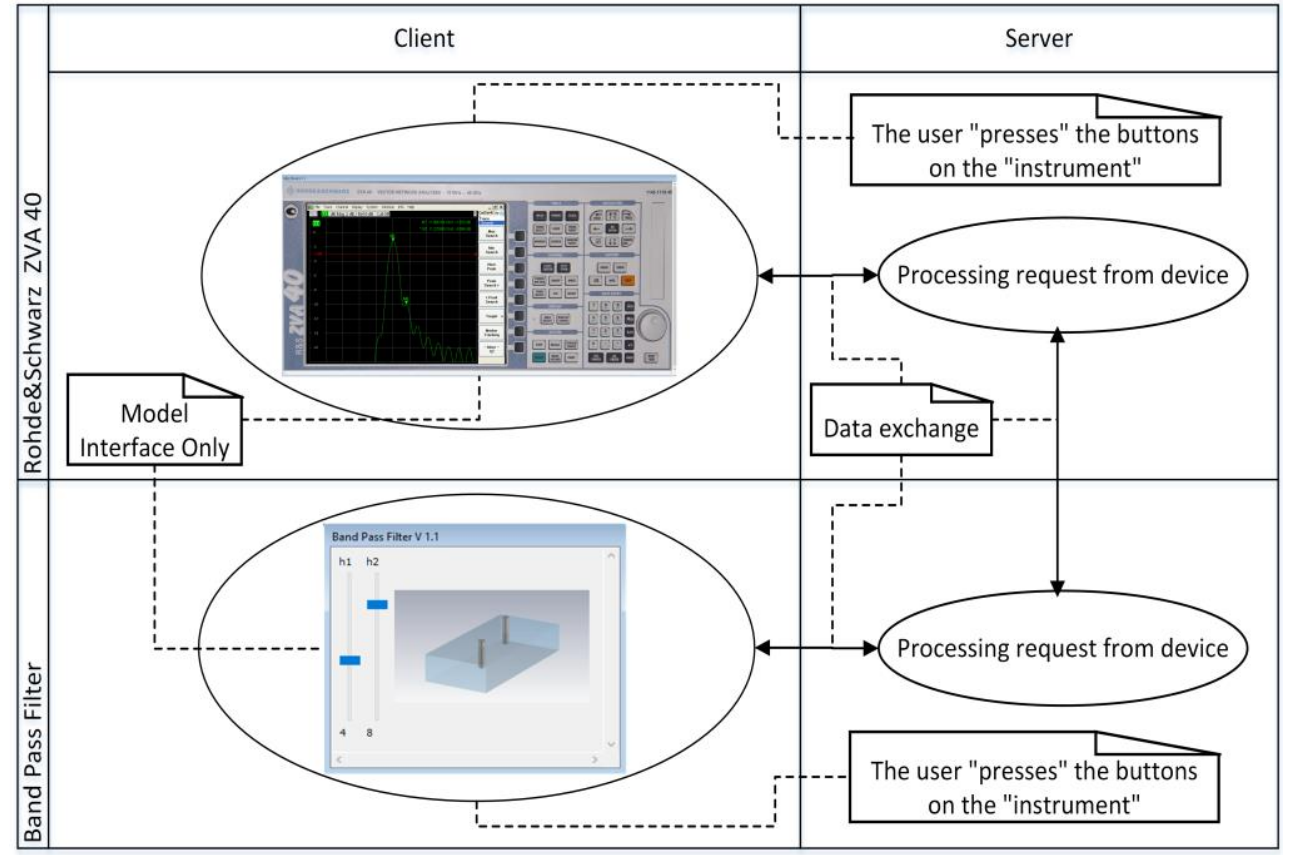

Fig. 2. Interaction of device models in a virtual laboratory.

As a result of designing, the autonomous laboratory is divided into two parts. The client part depends on the operating system (Windows, Android) and is installed by the user on its device. And the server part is developed on MSVS2015 and installed on a computer running Windows and connected to the Internet.

\section{Interaction of modules and the data exchange protocol}

Using the example of implementing a virtual laboratory with remote access, let us consider the interaction of modules and the data exchange protocol between client-server components. 
After installation of the client part on the device (currently is compatible with Windows OS only), the user can start performing the laboratory work. Having launched the application, the user will be offered to pass authorization. Then a timestamp packet (QTime, the current time on the user's device which is used to control and exclude packet repetition) including user login and password is sent to the server using TCP/IP protocol. After receiving the information, the server authenticates the user and determines the list of works available for execution.

When the test is successfully completed, the packet is returned to the client. The packet contains a QTime timestamp, a GUID session number (used to later identify packets), the number of laboratory works available to the user (NumWork) and the list of works (NameWork and its unique IdWork number). Otherwise, a GUID session number 1 is returned and the access is denied. If the server responds positively, the client application displays a list of available lab works and offers the user to start any of them. This interaction process is briefly shown in Fig. 3.

After running the laboratory work, the server receives a request to obtain the necessary data for drawing a measurement device on the screen (for example, a vector network analyzer ZVA-40). In these cases, the Client sends a request (Tables 1) and waits for a response from the Server (Tables 2). After receiving the data, the Client processes them, creates an array of values, indexed by frequency, and redraws the AFC of the device under study. A similar exchange of packets also takes place when it is necessary to obtain data for redrawing the screen of visualizing the amplitude-frequency characteristic of the device under study (Fig. 3). Next, the user can carry out all necessary measurements or select the operation mode of the vector network analyzer by "pressing" the corresponding buttons on the "real" instrument panel.

The proposed protocol for the interaction of devices should provide high speed data transmission and guaranteed delivery.

Table 1. Packet from client to server.

\begin{tabular}{|l|l|l|}
\hline Field name & Type and length, bytes & Function \\
\hline QTime & char, 4 & timestamp \\
\hline GUID & unsigned int, 4 & unique session number \\
\hline IdWork & unsigned int, 2 & unique lab work number \\
\hline NumMess & unsigned int, 2 & index number of the message \\
\hline fBeg & double, 8 & initial amplitude frequency response \\
\hline fEnd & double, 8 & initial amplitude frequency response \\
\hline LWin & unsigned int, 2 & amplitude response points \\
\hline
\end{tabular}

Table 2. Server response to client request.

\begin{tabular}{|l|l|l|}
\hline Field name & Type and length, bytes & Function \\
\hline QTime & char, 4 & timestamp \\
\hline GUID & unsigned int, 4 & unique session number \\
\hline IdWork & unsigned int, 2 & unique lab work number \\
\hline NumMess & unsigned int, 2 & index number of the message \\
\hline LWin & unsigned int, 2 & values array size \\
\hline ArrS & float, 4 & data array for plotting AFC \\
\hline
\end{tabular}




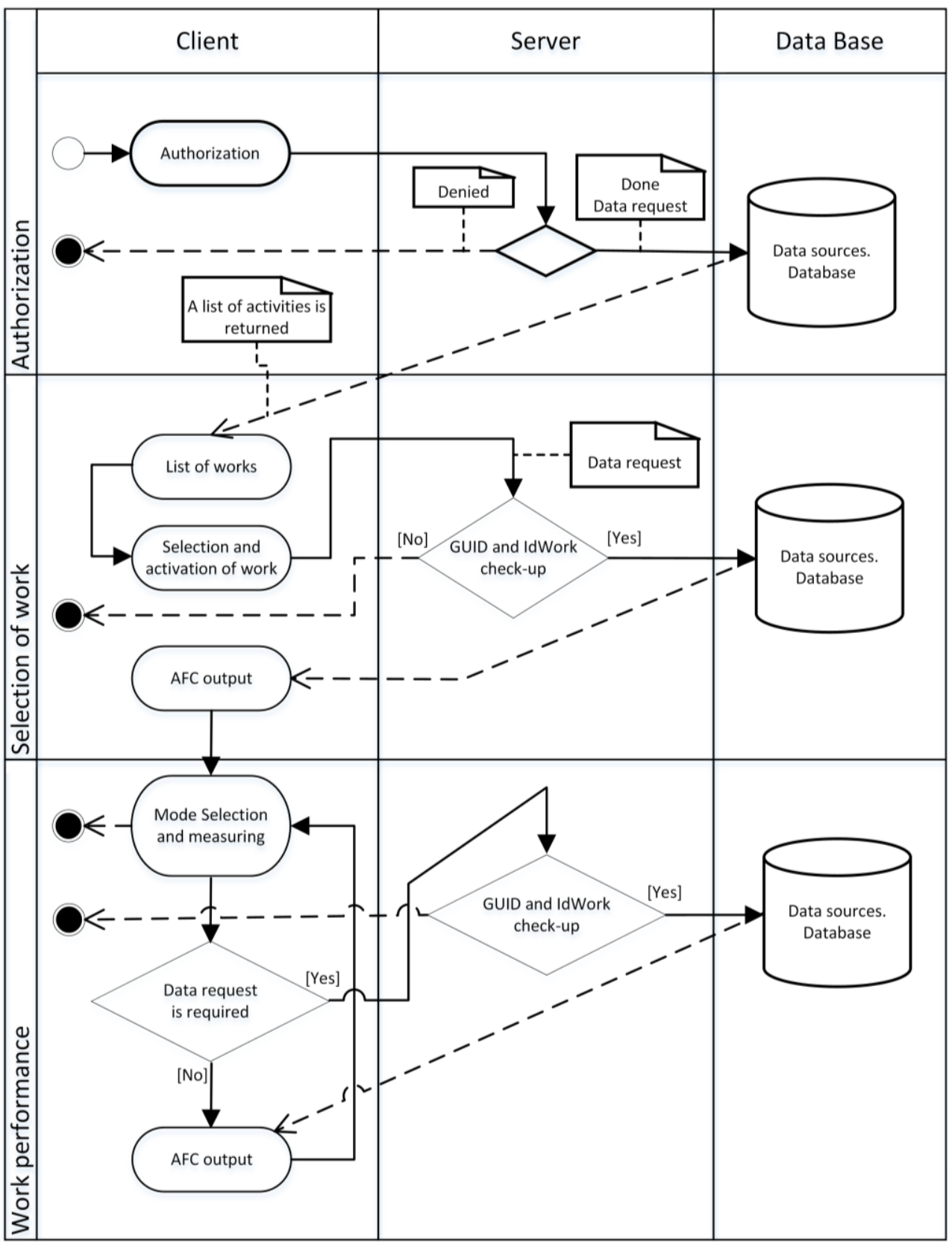

Fig. 3. Client-server interaction diagram.

\section{Conclusions}

As a result, the approach of upgrading an autonomous virtual laboratory into a laboratory with remote access was developed and tested. The created remote virtual laboratory was implemented and tested in the educational process at the Physical Faculty of Southern Federal University. Virtual laboratory with remote access were tested as part of Bachelor's degree program which includes the workshop "Microwave Measurements Devices". 
According to the results of the survey among students, most of them noted that performing virtual lab works clearly shows the essence of physical processes and the operation principle of the studied microwave devices. They also pointed out that virtual measuring instruments have almost full similarity with real devices terms of the user interface well as functioning and that virtual lab works allowed them to understand and master the principle of measurement using a variety of microwave measurement devices. All students as a whole were satisfied with the learning process in the remote access mode. Based on this, it can be stated that the developed laboratory with remote access provides students with an understandable and convenient virtual online resource to study devices and microwave devices. The proposed virtual laboratory provides a virtual tool for experimental research without the need to access real expensive measuring equipment. The results of laboratory tests in the educational process show that this laboratory is a convenient learning tool for conducting experimental studies that reduce the time needed to study real equipment in the laboratory. Remote virtual laboratory increases student interest in learning and deepens their understanding of the fundamental physics necessary for the operation of microwave devices. This can help students to understand and master the methods of making measurements in the microwave range, thereby improving the efficiency of teaching process, and gaining skills of independent work with real laboratory equipment. Consequently, students "lose their fear" before making measurements on real expensive equipment. Thus, we developed and implemented a virtual laboratory software package with remote access and created computer models of measurement equipment with a realistic interface simulating the operation of real measurement devices. The server software of the virtual laboratory is easily upgraded and expandable. The developed software package can be used for distance learning and is intended to replace real expensive measuring equipment. It also allows users to gain measurement skills on sophisticated hightech equipment without fear of technical failure.

\section{References}

1. S. Akrouf, A. Merabet, A. Maza, D. Boubetra, D., L. Selmani, A. Boubetra, N. Mouhoub, Web Services for Virtual Simulation, International Journal of Online Engineering, 5, pp.9-11 (2014)

2. Y.A. Daineko, M.T. Ipalakova, Z.Z. Bolatov, Employing information technologies based on.NET XNA framework for developing a virtual physical laboratory with elements of $3 D$ computer modelling, Programming and Computer Software, 3, pp. 161171 (2017)

3. S. Sanz, M. Iskander, L. Yu, Development of an Interactive Multimedia Module on Antenna Theory and Design, Computer Applications in Engineering Education, 1, pp. 11-16 (2000)

4. D.S. Gubsky, V.V. Zemlyakov, Advanced microwave equipment simulator for engineering education, International Journal of Electrical Engineering Education, 1, pp. 92-101 (2019)

5. Y.-H. Liu, Y.-T. He, D. Tian, R.-L. Fan, L. Yao, A web-based virtual laboratory for SHRIMP, Computer Applications in Engineering Education, 5, pp. 1493-1506 (2018)

6. X. Lu, D. Tian, Y.-T. He, L. Yao, R.-L. Fan, A Web-Based Virtual Laboratory For Electron Probe Microanalysis, Computer Applications in Engineering Education, 4, pp. 489-498 (2015)

7. A. Tekin, F. Ata, M. Gokbulut, Remote Control Laboratory for DSP-Controlled Induction Motor Drives, Computer Applications in Engineering Education, 4, pp. 702$712(2012)$ 
8. C. Bayilmis, Development of a Web-Based Educational Interface Using MATLAB Builder NE With Web Figure for Digital Modulation Techniques, Computer Applications in Engineering Education, 4, pp. 604-610 (2012)

9. S. Fuada, A. Salman, Virtual Labs of Analog Oscillator, International Journal of Online Engineering, 8, pp. 31-37 (2016)

10. J.L. Gómez-Tornero, D. Cañete-Rebenaque, F.D. Quesada-Pereira, A. ÁlvarezMelcón, Interactive lab to learn radio astronomy, microwave \& antenna engineering at the Technical University of Cartagena (Spain), International Journal of Online Engineering, 1, pp. 10-18 (2011)

11. A. Kalantzopoulos, E. Zigouris, Online Laboratory Sessions in System Design with DSPs using the R-DSP Lab, International Journal of Online Engineering, 4, pp. 4-12 (2014)

12. I. Titov, A. Glotov, A. Yelizarov, V. Petrov, Standartization Use Case of Solid-State Laser Lab and RF\&Microwave Amplifier Remote and Virtual Laboratories at Labicom, International Journal of Online Engineering, 9, pp. 47-51 (2016)

13. R. Pastor, A.C. Caminero, D. Sánchez, R.H.S. Ros, A. Robles-Gómez, L. Tobarra, Laboratories as a Service (LaaS): Using Cloud Technologies in the Field of Education, Journal of Universal Computer Science, 14, pp. 2112-2126 (2013)

14. E Fabregas, G. Farias, S. Dormido-Canto, S. Dormido, F. Esquembre, Developing a remote laboratory for engineering education, Computers and Education, 2, pp. 16861697 (2011)

15. L.F.Z. Rivera, M.M.L. Petrie, Models of Collaborative Remote Laboratories and Integration with Learning Environments, International Journal of Online Engineering, 9, pp. 14-21 (2016)

16. D.S. Gubsky, A.D. Gubsky, A.B. Kleshchenkov, Development A Computer Model Of Microstrip Filter For Virtual Laboratory, The International Scientific Conference Radiation and Scattering of Electromagnetic Waves (RSEMW), June 26-30, Gelendzhik, Russia, pp. 258-260 (2017)

17. D.S. Gubsky, V.V. Zemlyakov, D.V.Lonkina, Virtual Laboratory for Microwave Engineers Education, Progress In Electromagnetics Research Symposium (PIERS), Shanghai, China, Aug 8-11, pp. 3451-3454 (2016)

18. D.S. Gubsky, A.B. Kleschenkov, I.V. Mamay, Virtual laboratory for microwave measurements, Computer Applications in Engineering Education, (to be published) (2019) 